\title{
Specific heat of the quantum Heisenberg antiferromagnet by a renormalization-group approach
}

\author{
A. Drzewiński* and R. Dekeyser \\ Instituut voor Theoretische Fysica, Katholieke Universiteit Leuven, Celestijnenlaan 200D, B-3001 Leuven, Belgium
}

(Received 8 July 1996)

\begin{abstract}
The finite-temperature renormalization scheme has been applied to the antiferromagnetic Heisenberg model on the triangular lattice with inhomogeneous bonds (with the kagomé lattice as a limiting case). In order to preserve the isotropy of the Hamiltonian, this method was modified by using the eigenvectors of the so-called "parity" operator. This allows to analyze the model which corresponds to some experiments of a ${ }^{3} \mathrm{He}$ layer adsorbed on graphite in the $\mathrm{mK}$ temperature region. The appearence of more than one maximum in the specific heat is discussed as a function of the imposed inhomogeneity. [S0163-1829(96)08245-8]
\end{abstract}

\section{INTRODUCTION}

Over the last few years the density matrix renormalization group (DMRG) introduced by White ${ }^{1}$ has revived the interest of physicists in the ground-state renormalization. This method, like the original truncation method, ${ }^{2-4}$ can be applied to quantum many-body Hamiltonians at $T=0$ and leads often to amazingly accurate results for the ground-state energy, excited states or the spin-spin correlation functions. However, the DMRG scheme has rather no special advantage over the truncation method in calculating critical points and their exponents. ${ }^{5-7}$ The main difference between both methods consists in the choice of the cell states that are kept in successive steps of a renormalization-group (RG) transformation. The DMRG approach is based on keeping those states, which are especially appropriate for representing the ground state of a larger system (a so-called superblock), instead of lowest-energy states of the cell usually kept in the standard truncation method. In both cases the choice of kept states should guarantee that the symmetries of the original problem are preserved under the RG transformation, which, e.g., should not turn a model with isotropic interactions into an anisotropic model. For a finite-temperature RG, where all states have to be taken into account, it is impossible from the mathematical point of view to provide the ideal RG transformation. Therefore we ought to look for an optimal assignment between original and effective states, keeping in mind the symmetries of our problem.

This is why the success of the DMRG approach encouraged us to reconsider the finite-temperature RG transformations previously proposed by Stella et al. ${ }^{8,9}$ This method has turned out to be fruitful for the investigation of thermodynamic properties of the quantum Heisenberg antiferromagnet (HAF).

The general form of our Hamiltonian is

$$
\mathcal{H}=J \sum_{\langle i, j\rangle} \overrightarrow{S_{i}} \cdot \vec{S}_{j}
$$

where the variables $S_{i}^{m}(m=x, y, z)$ are the usual spin-1/2 operators represented by Pauli matrices at the $i$ th site. Since the frustrated spin systems have provided an attractive setting for attacking problems concerning both the ground state and the thermodynamic properties, we have decided to con- sider the HAF on the triangular lattice with inhomogeneous bonds. With the aim of describing the introduced inhomogeneity of interactions we distinguish one triangular sublattice ( $\bigcirc$ ) from three others (O). As we can see in Fig. 1, this introduces a division of the bond interactions in two sets $\left(J \rightarrow J_{K}, J_{L}\right)$. Considering the case with both parameters nonnegative, where $J_{K} \geqslant J_{L}$, we investigate an inhomogeneous lattice whose limits coincide with (i) the triangular lattice for $J_{K}=J_{L}$ and (ii) the kagomé lattice for $J_{K}>0$ and $J_{L}=0$.

For a long time these models have been suspected to possess a magnetically disordered ground state on account of geometrical frustration. However, most numerical investigations have indicated ${ }^{10}$ that the HAF on the triangular lattice remains ordered at $T=0$. The next candidate was the HAF on the kagomé lattice, which is highly frustrated, and has a low coordination number and a large number of classically degenerate states. Numerical studies using series expansions ${ }^{11}$ and an exact diagonalization ${ }^{12}$ have indicated that the ground state of the HAF on the kagomé lattice has no long-range magnetic order.



FIG. 1. The triangular lattice with inhomogeneous interactions. 
Recent significant interest in the HAF on the latter lattice originates from experiments of a ${ }^{3} \mathrm{He}$ layer adsorbed on graphite in the $\mathrm{mK}$ temperature region. Apart from the standard higher-temperature maximum, an additional sharp peak of the specific heat was observed at a coverage $\rho \cong 0.18$ atoms $/ \AA^{2}$, where the second layer is just filled. The authors of those experiments ${ }^{13}$ proposed that there is a transition between the fluid phase and a registered solid. At higher temperatures there is a typical rounded maximum. Elser ${ }^{14}$ assumed that the atoms on the first layer form a highly compressed triangular lattice with the $\sqrt{3} \times \sqrt{3}$ structure that determines the $\sqrt{7} \times \sqrt{7}$ structure on the second layer. A quarter of the second-layer atoms (located directly above the first-layer atoms) have a less favorable potential energy than the other atoms (located "between" the first layer atoms). For details we refer to Fig. 1 in Ref. 14. This distinction decomposes the second layer into a kagomé net (three sublattices) with exchange constants $J_{K}$ and the remaining sublattice interacting weakly with the other atoms (exchange constants $J_{L}$ ), where $J_{K} \gg J_{L}$.

The existence of the second peak is also supported by a lack of entropy in the above experiments, when the total change in entropy per site between $T=\infty$ and the extrapolated value for $T=0$ is calculated. When three sublattices form the $\sqrt{7} \times \sqrt{7}$ structure, the fourth-sublattice atoms are almost free and half the missing entropy has to come from them. The rest of the missing entropy is to be supplied just by the low-temperature peak.

The numerical diagonalization of finite clusters ${ }^{15}$ and Monte Carlo simulations ${ }^{16}$ seem to confirm the existence of the second peak at low temperatures. Using high-temperature expansions ${ }^{15}$ and a numerical technique called the forcedoscillator method, ${ }^{17}$ contradictory results were found. That is why it is tempting to use a nonperturbative method as the RG one, which has often been applied to the investigation of properties of interacting many-body systems. In a previous paper a version of the RG method for the Ising and $X Y$ models on the inhomogeneous triangular lattice was presented. ${ }^{18}$ In the present paper we now apply it to the HAF system.

\section{RG TRANSFORMATION}

In the Introduction we have started our considerations from the truncation method - a ground-state version of the RG approach. ${ }^{19}$ Therefore, coming to finite temperatures we prefer such a version of the finite-temperature RG, which gives the truncation method as its zero-temperature limit. Such an approach has been presented by Stella et al., ${ }^{9}$ among others also for the models with a degenerate cell ground state as is the case in the HAF model. Let us first consider the homogeneous case.

\section{A. Assignment of the cell states on the triangular lattice}

During the RG transformation the lattice is divided into cells and the Hamiltonian into an intracell part $\mathcal{H}_{0}$ and an intercell coupling $\mathcal{V}: \mathcal{H}=\mathcal{H}_{0}+\mathcal{V}$. When we choose, for example, the hexagonal cell with seven sites (see Fig. 1), each cell has 128 states belonging to separate subspaces of the cell Hamiltonian's matrix $\mathcal{H}_{0 \alpha}$. They can, e.g., be classified ac- cording to the eigenvalues of the total $z$ component of the spin, $\hat{S}^{z}=\sum_{i} S_{i}^{z}$ ( $i$ denotes the cell sites), because $\hat{S}^{z}$ commutes with the cell Hamiltonian $\left[\mathcal{H}_{0 \alpha}, \hat{S}_{\alpha}^{z}\right]=0$. Due to the spin-space symmetry of the HAF, these states appear in couples (Kramers doublets): If an eigenvector belongs, e.g., to the subspace with the eigenvalue $S^{z}=+1$, there exists a partner belonging to the subspace with the eigenvalue $S^{z}=-1$. In order to construct the RG transformation we have to divide all eigenvectors into two groups, which is necessary in order to obtain the effective Hamiltonian as a spin-1/2 Hamiltonian at each iteration. In the first place we know that eigenvectors for each Kramers doublet should be separated. Moreover, there is no point in splitting eigenvectors belonging to the same subspace of the $S^{z}$, since this would introduce spurious anisotropies in the $x y$ plane. Keeping this in mind, Stella et al. divided the subspaces according to the sign of the eigenvalues of the $\hat{S}^{z}$ operator. The $\alpha$ th cell-Hamiltonian eigenvectors from the subspaces with $S^{z}=+1,+3,+5,+7$ constitute the first group $\left|\sigma_{\alpha}^{z}=+1, \tau_{\alpha}\right\rangle$, where $\tau=1, \ldots, 64$. At the same time all remaining eigenvectors $\left(S^{z}=-1,-3,-5,-7\right)$ form the second group $\left|\sigma_{\alpha}^{z}=-1, \tau_{\alpha}\right\rangle$. The symbol $\sigma_{\alpha}^{z}$ distinguishes both groups and can be identified with the eigenvalue of the $z$ component of the effective cell spin.

Now we can define the RG transformation leading to an effective spin Hamiltonian $\mathcal{H}^{\prime}$ :

$$
\left\langle\sigma_{\alpha}^{z}\left|e^{-\beta^{\prime} \mathcal{H}^{\prime}}\right| \sigma_{\alpha}^{\prime z}\right\rangle=\operatorname{Tr}_{\tau_{\alpha}}\left\langle\sigma_{\alpha}^{z}, \tau_{\alpha}\left|e^{-\beta \mathcal{H}}\right| \sigma_{\alpha}^{\prime z}, \tau_{\alpha}\right\rangle .
$$

Since we are not able to solve this equation exactly, we have to use an approximation. As it has been presented in Ref. 9, using the Feynman identity, we can expand both sides of Eq. (2) in powers of $\mathcal{V}$. Constructing the approximate form of the RG transformation up to the first order, we obtain in every step a contribution to the dimensionless free energy as a function of cell energy levels $E(\tau)$ :

$$
\Delta \widetilde{f}=\beta E_{0}-A
$$

where

$$
A=\ln \left(\frac{\tilde{n}}{2}+\sum_{\tau=\widetilde{n} / 2+1}^{n / 2} e^{-\beta\left[E(\tau)-E_{0}\right]}\right)
$$

The symbol $n$ denotes the number of cell states, and $\tilde{n}$ denotes the degeneracy of the cell ground state $E_{0}$. By repeating the RG transformation, the dimensionless free energy is calculated iteratively by accumulating the cell energies according to the formula

$$
\widetilde{f}=\sum_{m=0}^{\infty} \frac{\Delta \widetilde{f}^{(m)}}{b^{m+1}},
$$

where $b$ is the number of sites in a cell and $m$ enumerates the successive RG steps.

The interactions between adjacent cells (e.g., $\alpha$ and $\alpha^{\prime}$ ) are also reconstructed and the contribution (here only from one original bond) to the RG transformation has the following form for its matrix elements: 


$$
\begin{aligned}
& K^{\prime}\left\langle\sigma_{\alpha}^{z}\left|\sigma_{\alpha}^{m}\right| \sigma_{\alpha}^{\prime z}\right\rangle \otimes\left\langle\sigma_{\alpha^{\prime}}^{z}\left|\sigma_{\alpha^{\prime}}^{m}\right|{\sigma^{\prime}}_{\alpha^{\prime}}^{\prime z}\right\rangle \\
& =K e^{-2 A}\left(\sum_{\tau_{\alpha}, \tau_{\alpha^{\prime}}=1}^{\tilde{n} / 2} B_{\tau_{\alpha}} B_{\tau_{\alpha^{\prime}}}\right. \\
& \quad+\sum_{\tau_{\alpha}=1}^{\tilde{n} / 2} \sum_{\tau_{\alpha^{\prime}}=\tilde{n} / 2+1}^{n / 2} e^{-\beta\left[E\left(\tau_{\alpha^{\prime}}\right)-E_{0}\right]} B_{\tau_{\alpha}} B_{\tau_{\alpha^{\prime}}} \\
& \quad+\sum_{\tau_{\alpha}=\tilde{n} / 2+1}^{n / 2} \sum_{\tau_{\alpha^{\prime}}=1}^{n / 2} e^{-\beta\left[E\left(\tau_{\alpha}\right)-E_{0}\right]} B_{\tau_{\alpha}} B_{\tau_{\alpha^{\prime}}} \\
& \quad+\sum_{\tau_{\alpha}, \tau_{\alpha^{\prime}}=\tilde{n} / 2+1}^{n / 2} e^{-\beta\left[E\left(\tau_{\alpha}\right)+E\left(\tau_{\alpha^{\prime}}\right)-2 E_{0}\right]} B_{\tau_{\alpha}} B_{\tau_{\alpha^{\prime}}},
\end{aligned}
$$

where $\quad B_{\tau_{\alpha}}=\left\langle\sigma_{\alpha}^{z}, \tau_{\alpha}\left|S_{\alpha i}^{m}\right| \sigma_{\alpha}^{\prime z}, \tau_{\alpha}\right\rangle \quad(m=x, y, z \quad$ and $i=1, \ldots, 7$ indices are omitted here in $\left.B_{\tau_{\alpha}}\right)$ and $K=\beta J$. For the triangular lattice split into hexagonal cells, there are three original bonds between adjacent cells. So three of the above contributions give us the recursion relations for the effective interactions $K^{\prime}=K^{\prime}(K)$.

As has been presented, this method allows us to make consistent approximate calculations of the free energy at all temperatures. ${ }^{9}$ For example, for the $X Y$ chain, where exact results are available, the free energy and the specific heat calculations are quite successful, already in the first-order RG scheme, where both sides of Eq. (2) are expanded up to first order in $\mathcal{V}$ (or $\mathcal{V}^{\prime}$ ). It is worth mentioning that such calculations would be more complicated in the presence of a phase transition, but such a case is out of our interest in the present paper.

Unfortunately, when we perform this calculation for the antiferromagnetic Heisenberg chain, the proposed RG scheme introduces an artificial anisotropy in the effective interactions: The effective $J^{\prime}$ in front of the terms $\sigma_{\alpha}^{x} \sigma_{\alpha^{\prime}}^{x}$ and $\sigma_{\alpha}^{y} \sigma_{\alpha^{\prime}}^{y}$ differs from $J^{\prime}$ in front of the term $\sigma_{\alpha}^{z} \sigma_{\alpha^{\prime}}^{z}$. In order to avoid this discrepancy we decided to reconsider the assignment proposed by Stella et al.

In order to assign the cell states to the site states we have to find basic properties of the cell states which would correspond best to the site-states properties. Let us first consider a (effective) site on whose spin space the cell states are projected. For the common matrix representation the eigenvectors of the Pauli matrix $\sigma^{z}$ have the following form: $|+\rangle_{z}=[1,0]$ and $|-\rangle_{z}=[0,1]$. At the same time $| \pm\rangle_{x}=[1, \pm 1]$ are the eigenvectors of the Pauli matrix $\sigma^{x}$ belonging to the eigenvalues \pm 1 , respectively. Their basic properties may be described by the fact that $|+\rangle_{z}=\left(|+\rangle_{x}+|-\rangle_{x}\right) / 2$ remains invariant under a reversal of the $x$ component of the spin, whereas $|-\rangle_{z}$ $=\left(|+\rangle_{x}-|-\rangle_{x}\right) / 2$ changes sign under such a reversal. The operator $\sigma^{x}$ can indeed be interpreted as the operator that exchanges the states with $S^{z}= \pm 1$. Of course, because of the symmetry of the problem, we could consider any pair of the spin components.

If we now look to the spin states of a triangular cell (for a simplicity we do not analyze here the hexagonal cell), we can split them according to the eigenvalue of the total $z$ component of the spin. We have here the eigenstates

$$
\begin{aligned}
& v_{+3}=|+\rangle \otimes|+\rangle \otimes|+\rangle, \\
& v_{-3}=|-\rangle \otimes|-\rangle \otimes|-\rangle .
\end{aligned}
$$

Next we have also two 3-dimensional subspaces $v_{+1}$ and $v_{-1}$ corresponding to the eigenvalues \pm 1 . The subspace $v_{+1}$, e.g., is spanned by the states

$$
|+\rangle \otimes|+\rangle \otimes|-\rangle, \quad|+\rangle \otimes|-\rangle \otimes|+\rangle, \quad|-\rangle \otimes|+\rangle \otimes|+\rangle .
$$

Stella et al. divided these four subspaces according to the sign of the eigenvalues $S^{z}$, which automatically distinguished the $z$ coordinate with respect to the others; it is also the origin of the artificially introduced anisotropy. In our opinion it is better to take into account the inversion of the spin space. Under a reversal of the $x$ components of all spins, the states of the $v_{+1}$ subspace get clearly multiplied with a factor -1 . It is therefore more natural to group this subspace together with $v_{-3}$ (which is also multiplied by -1 ) and to assign the resulting four-dimensional subspace to the eigenvalue $\sigma_{\alpha}^{z}=-1$. In a similar way, $v_{+3}$ and $v_{-1}$ are assigned to the eigenvalue +1 for the $z$ component of the cell spin.

The above division of the cell states can be presented in another way. We can notice that the Hamiltonian of the system has a simple invariance with respect to a simultaneous change in sign of all $S_{i}^{x}$ and $S_{i}^{y}$ while keeping the $S_{i}^{z}$ invariant (such that the commutation relations are not affected). ${ }^{5}$ Of course, because $\mathcal{H}$ is invariant under any permutation of the spin coordinates, there are two additional invariances, where $S_{i}^{x}$ and $S_{i}^{y}$ are kept invariant, respectively. As above we consider only the first case in detail. The invariance mentioned above corresponds to the relation $\left[\mathcal{H}_{0 \alpha}, \hat{P}_{\alpha}^{z}\right]=0$ (see in Ref. 3 ), where $\hat{P}_{\alpha}^{z}=\sigma_{\alpha 1}^{z} \otimes \cdots \otimes \sigma_{\alpha p}^{z}$ ( $p$ is the number of sites in the $\alpha$ th cell). As we can see, the eigenstates of the total $z$ component $\hat{S}^{z}$ are also eigenstates of the $\hat{P}^{z}$ operator.

In general let us call $\hat{P}_{\alpha}^{m}(m=x, y, z)$ the "parity" operators because their eigenvalues are \pm 1 . This duality can split the cell eigenstates in a natural way. For the seven-site cell it means that the subspaces with $S^{z}=+7,+3,-1,-5$ will belong to the first group and the others to the second one. What is more, the cell operators $\hat{P}_{\alpha}^{m}$ fulfill the same relations, e.g., $\hat{P}_{\alpha}^{z} \hat{P}_{\alpha}^{x}=i \hat{P}_{\alpha}^{y}$ as the site operators $\sigma_{i}^{m}$ (up to the common factor \pm 1 or $\pm i$, which depends on the number of sites in a cell), which is not true for the total $m$ component spin operators. It means that the "parity" operator properties correspond in a better way to the site spin- $1 / 2$ operators than the total spin operators. However, both types of cell operators $\left(\hat{P}_{\alpha}^{m}\right.$ and $\hat{S}_{\alpha}^{m}$ ) fulfill the full commutator relations, e.g., $\left[\hat{S}_{\alpha}^{z}, \hat{S}_{\alpha}^{x}\right]=2 i \hat{S}_{\alpha}^{y}$.

We have checked that in fact such a division according to the eigenvalues of the "parity" operators conserves the symmetrical form of the Hamiltonian with respect to the spin coordinates in each step of the RG transformation at least in a first order procedure.

\section{B. Inhomogeneous interactions}

As we have mentioned, the region of the Hamiltonian parameters where $J_{K} \gg J_{L}$ is the most important for our investigations. Therefore we have decided to make use of the scheme for the inhomogeneous triangular lattice, which was 
already applied for the Ising and the $X Y$ models in the ferromagnetic case. ${ }^{18}$ In order to conserve the four-sublattice symmetry of our bond division we use the seven-site cells. As we can see in Fig. 1, such a choice implies the existence of two types of cells, which we have named the $K$ cells and the $L$ cells. Because our calculations have been done to first order in $\mathcal{V}$, only the first neighbors still interact after the RG transformation. Comparing with the triangular-lattice case, we can see that now the recursion relations have a more complex form

$$
\begin{aligned}
& K^{\prime}=f_{1} K+2 f_{2} L, \\
& L^{\prime}=2 f_{3} K+f_{4} L,
\end{aligned}
$$

where the $f_{j}$ are defined through the partial expectation values

$$
\begin{aligned}
& \left\langle S_{2 \alpha_{K}}^{m} S_{2 \alpha_{K}^{\prime}}^{m}\right\rangle=f_{1} \sigma_{\alpha_{K}}^{m} \sigma_{\alpha_{K}^{\prime}}^{m}, \quad\left\langle S_{2 \alpha_{K}}^{m} S_{4 \alpha_{K}^{\prime}}^{m}\right\rangle=f_{2} \sigma_{\alpha_{K}}^{m} \sigma_{\alpha_{K}^{\prime}}^{m}, \\
& \left\langle S_{2 \alpha_{L}}^{m} S_{2 \alpha_{K}^{\prime}}^{m}\right\rangle=f_{3} \sigma_{\alpha_{L}}^{m} \sigma_{\alpha_{K}^{\prime}}^{m}, \quad\left\langle S_{2 \alpha_{L}}^{m} S_{4 \alpha_{K}^{\prime}}^{m}\right\rangle=f_{4} \sigma_{\alpha_{L}}^{m} \sigma_{\alpha_{K}^{\prime}}^{m},
\end{aligned}
$$

where $i \alpha_{K}$ and $i \alpha_{L}(i=1, \ldots, 7)$ denote the sites in the $K$ and $L$ cells. The factor $\beta$ has been absorbed in the Hamiltonian: $K=\beta J_{K}$ and $L=\beta J_{L}$. It is worthwhile to notice that every bracket above factorizes in terms connected with separate cells. In order to use Eqs. (3) and (4) for calculating the free energy, we should only keep in mind that three-quarters of the contribution in each step comes from the $K$ cells, whereas the rest comes from the $L$ cells. Using the free energy, we will be able to calculate the temperature dependence of the specific heat for different ratios $K / L$.

We have to observe, however, that the trick with the inhomogeneous bonds should be applied cautiously in order to avoid pathological cases. For example, if one distinguishes two triangular sublattices from the two others by setting the interactions between these two groups at zero, a set of noninteracting chains would be obtained. It is known that for the $d=1$ Ising model there is no phase transition at finite temperatures. When we adopt our trick for the Ising model on this inhomogeneous triangular lattice, a ferromagnetic fixed point (connected with the homogeneous triangular lattice) appears, which attracts points from its critical line. Because the critical line crosses also the region corresponding to separated chains, we find also here a phase transition, which is, of course, false. Although it has been performed ${ }^{20}$ for another version of the RG transformation, ${ }^{21}$ the same spurious effect is likely to be obtained for our transformation too.

We believe that our trick with the inhomogeneous bonds can be applied for investigating the region between the pure triangular and the pure kagomé lattices, although the latter limit is less trustworthy.

Some doubts can also be raised, when the antiferromagnetic order is considered. Because we do not know the ground state of this ordering, it is not clear how well it is conserved by our particular choice of cells. Knowledge of this ground state would be very important for the investiga- tion of a phase transition. We believe that here, when the free energy is calculated, it is less relevant and our choice of cells is correct.

\section{Zeroth-order approach}

It has been clear that, in order to find the thermodynamic properties, we have to take into account all cell states. For calculating low-temperature properties, however, one could treat the cell states with an energy above the cell ground states in a more approximate way by taking them to be decoupled between different cells. This procedure has been applied by Hirsch and José. ${ }^{22}$ to the $d=1$ HAF and reasonable results were obtained. As far as the recursion relations are concerned, all higher states are neglected and only the cell ground states are kept. Then only the first term in the righthand side of Eq. (5) survives, the coefficient $e^{-2 A}$ in Eq. (5) goes to the simple form $(2 / \tilde{n})^{2}$ and the factor $\beta$ cancels on both sides of Eq. (5), leading to the result

$$
J^{\prime}=\left(\frac{2}{\tilde{n}}\right)^{2} J \sum_{\tau_{\alpha}, \tau_{\alpha^{\prime}}}^{\tilde{n} / 2} B_{\tau_{\alpha}} B_{\tau_{\alpha^{\prime}}}
$$

If there is only one ground-state doublet $(\tilde{n}=2)$, the above equation is equivalent to the formula in the standard truncation method. As we can see, in the zeroth-order approximation the temperature is like a parameter, which is not subject to renormalization.

To make this method applicable for studying finitetemperature properties of the system, we take into account all cell states when the free energy is calculated. Computing in the zeroth order (in the intercell interactions $\mathcal{V}$ ) we obtain the following form of Eq. (3) for the free energy:

$$
\Delta f=E_{0}(\tau=1)-T \ln \left(1+\frac{Z_{h}}{Z_{l}}\right),
$$

where $Z_{l}$ and $Z_{h}$ are the cell partition functions for the ground-state doublet (with energy $E_{0}$ ) and for the remaining cell states, respectively. Hirsch and José ${ }^{22}$ considered the case when all remaining states have higher energies than $E_{0}(\tilde{n}=2$ here $)$. In the next section we present results for both orders of the approximation.

\section{SPECIFIC HEAT BEHAVIOR}

\section{A. Kagomé-like region}

As we have mentioned, the second-layer Hamiltonian is believed to have the Heisenberg form with two kinds of bonds on the triangular lattice $\left(J_{K}\right.$ and $\left.J_{L}\right)$. Because the magnetization measurements ${ }^{23}$ require that at least $J_{K}$ have the antiferromagnetic sign, we will investigate the region where both parameters are positive. An outlined microscopic explanation of the choice $J_{K} \gg J_{L}$ has been presented in the Introduction.

In the following calculations, the specific heat of the HAF is evaluated as the second derivative of the free energy. The (dimensionless) free energy per spin can be calculated by 


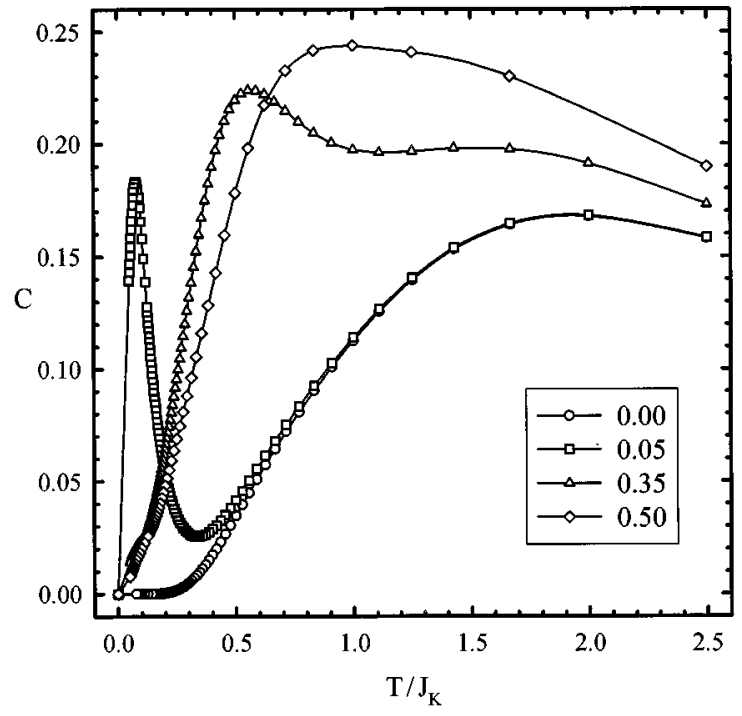

FIG. 2. The specific heat calculated for different bond ratios $J_{L} / J_{K}$.

iterating the renormalization process according to Eq. (3) or (8). The specific heat can be expressed in terms of the free energy per spin as

$$
C=-T \frac{\partial^{2} f}{\partial T^{2}}
$$

whereas for the dimensionless free energy we can use the formula

$$
C=K^{2} \frac{\partial^{2} \tilde{f}}{\partial K^{2}},
$$

where $K=\beta J$.

When the free energy is considered in zeroth order, it is easy to find the ground-state energy. For the pure kagomé lattice we have found the value $\epsilon_{0}=-1.392$ which can be compared with the result ${ }^{12}$ coming from a finite-cluster diagonalization $\epsilon_{0}=-1.752$.

Our first-order results for the specific heat are presented in Fig. 2 for the bond ratio $J_{L} / J_{K}=50 / 100,35 / 100,5 / 100$, and 0 , respectively. In the kagomé-like region zeroth- and firstorder approximations give qualitatively the same picture. For a relatively high ratio $J_{L} / J_{K}=0.5$ (and higher) there is a rounded maximum at higher temperatures. As usual this is connected with the equal probability of states in the infinitetemperature limit. Let us call this maximum the first peak (I peak). As the bond ratio descends the position of the first peak moves to lower temperatures and its height reduces slowly. The results for both approximations have been collected in Table I. At the same time the high-temperature slope is more and more flat. At a specific ratio (around 0.38) a second maximum appears, which will play the role of the high-temperature maximum from that moment on. Let us call this new maximum the second peak. As we continue to go down with the bond ratio, the position of the second peak will converge to the final position $T / J_{K} \simeq 1$.9. Simultaneously the shape of the first peak changes drastically: It
TABLE I. The positions and heights of the first peak in the specific heat as a function of the bond ratio $J_{L} / J_{K}$. Here and in Table II superscripts denote the order of the approximation.

\begin{tabular}{lcccc}
\hline \hline$J_{L} / J_{K}$ & $50 / 100$ & $35 / 100$ & $5 / 100$ & 0 \\
\hline$\left(T / J_{K}\right)^{(0)}$ & 1.01 & 0.583 & 0.081 & - \\
$C^{(0)}$ & 0.243 & 0.222 & 0.182 & - \\
$\left(T / J_{K}\right)^{(1)}$ & 0.962 & 0.57 & 0.078 & - \\
$C^{(1)}$ & 0.244 & 0.224 & 0.184 & - \\
\hline \hline
\end{tabular}

becomes very sharp and its position goes to zero temperature. In our opinion it seems reasonable to identify this peak with the additional low-temperature maximum of the specific heat, which was observed for the second ${ }^{3} \mathrm{He}$ layer.

In general maxima of the specific heat correspond to maxima of the density of states. When we use the RG transformation, which is based on a division of the lattice into cells, the specific-heat function has in first instance to be connected with the spectrum of energy levels for a cell. For the $K$ cell, when we consider the triangular lattice, there is only one ground-state doublet. As the bond ratio goes to zero the distances between this doublet and the next 11 doublets decrease until the ground state becomes 24-fold degenerate. For a small enough ratio the gaps decrease proportionally to the bond ratio. As can be expected also the position of the first peak moves here to zero temperature according to the same relation $\left(T / J_{K}\right)_{\text {Ipeak }} \sim J_{L} / J_{K}$. For the pure kagomé lattice the gaps between the ground state and the excited states of the $K$ cell are closed and the low-temperature peak disappears.

However, it is likely that if we choose larger cells, this gap may still be open for $J_{L} / J_{K}=0$. At the same time in the kagomé-like region the $L$ cell has the same ground-state doublet and its structure of energy levels seems not to affect the specific heat. For another lattice division we may have more types of cells affecting the specific heat. So these deficiencies of the RG approach are susceptible of influencing (e.g., suppressing) the low-temperature maximum in the specific heat, also for the pure kagomé lattice. The relation between its position and the bond ratio might then, e.g., look like $\left(T / J_{K}\right)_{\text {Ipeak }}=a+b\left(J_{L} / J_{K}\right)$, where $a$ and $b$ are some constants. Such an assumption would correspond to results of other papers, ${ }^{15,16}$ which also considered the kagomé lattice, and where the low-temperature peak was found. Nonetheless, we should remember that the second ${ }^{3} \mathrm{He}$ layers used in experiments seems to possess inhomogeneous bonds fulfilling the condition $J_{K} \gg J_{L}$.

Regarding the validity of our approximations for the kagomé lattice we can find in Table II a deviation (in height)

TABLE II. The positions and heights of the second peak in the specific heat as a function of the bond ratio $J_{L} / J_{K}$.

\begin{tabular}{lcccc}
\hline \hline$J_{L} / J_{K}$ & $50 / 100$ & $35 / 100$ & $5 / 100$ & 0 \\
\hline$\left(T / J_{K}\right)^{(0)}$ & - & 1.511 & 1.942 & 1.941 \\
$C^{(0)}$ & - & 0.199 & 0.168 & 0.224 \\
$\left(T / J_{K}\right)^{(1)}$ & - & 1.41 & 1.905 & 1.905 \\
$C^{(1)}$ & - & 0.198 & 0.168 & 0.168 \\
\hline \hline
\end{tabular}


for the second peak (in the zeroth order) with respect to the $J_{L} \neq 0$, due to the special value of $\tilde{n}$ at $J_{L}=0$ [see Eq. (7)]. This suggests that for the highly degenerate ground state of a cell the free-energy calculations are less trustworthy in zeroth order. The first-order calculations confirm the absence of the low-temperature maximum, but we should remember that at very low temperatures the results suffer from an additional inaccuracy connected with a very large initial $K$ (or $L)$ which results in a large number of significant RGtransformation steps.

\section{B. Triangularlike region}

In the previous subsection we discussed the behavior of the HAF in the region, which is believed to correspond to the nuclear-spin ordering in the adsorbed phases of ${ }^{3} \mathrm{He}$ on graphite. Because our method can be extended to the triangularlike region $\left(\frac{1}{2} J_{K}<J_{L} \leqslant J_{K}\right)$, it seemed interesting to perform also here calculations of the free energy.

The ground-state energy for the triangular lattice has the value $\epsilon_{0}=-1.82$. The series-expansion method gives as estimate for the ground-state energy ${ }^{11} \epsilon_{0} \simeq-2.21$ in this case. As we can see the deviation from the best approximation is here more or less the same as for the kagomé lattice.

As far as the specific heat is concerned, there is only one high-temperature maximum (the first peak) in the zeroth order. As the bond ratio decreases the maximum starts to flatten and it also shifts towards lower temperatures. For the first-order calculations we have found the same behavior (of the high-temperature maximum) with only small corrections for position and height $\left(T / J_{K} \simeq 1.59\right.$ and $C^{(1)} \simeq 0.3$, respectively). Unexpectedly another low-temperature maximum appears here around the value $J_{L} / J_{K} \sim 0.8$. When this ratio goes down, the additional peak goes to zero temperature, reducing its height. Around $J_{L} / J_{K} \sim 0.6$ we do not observe it anymore.

It is difficult to say if this peak is a spurious effect of the first-order calculations or if we have found a new structure in the energy levels. Because of complexity, we have not been able to extend our calculations to the second order. We can mention that if we stop to accumulate the contributions to the free energy after the RG first step, this is equivalent to the exact diagonalization on the seven-site cell (averaging over their two types). In that case we also observe the presence of an additional (broad) maximum for the region of $J_{L} / J_{K}$ mentioned above.

At the same time our first-order calculations suffer here also from another evidently spurious effect. That means that for very large $K$ and $L$ (low temperatures) the specific heat is slightly negative. As we have checked, the reason is the fact that second derivatives, like $\partial^{2} K^{\prime} / \partial K^{2}$, can be negative. Because these derivatives are extremely sensitive on the form of the recursion relations, it is very likely that the higherorder approximations would cancel this nonphysical behavior of the specific heat.

\section{CONCLUSIONS}

The aim of this paper has been to check the usefulness of the RG approach in studying the thermodynamic properties of a quantum spin system. We have chosen the quantum Heisenberg antiferromagnet on the inhomogeneous triangular lattice, because this model is believed to correspond to the interesting experiments of a ${ }^{3} \mathrm{He}$ layer adsorbed on graphite in the $\mathrm{mK}$ temperature region. The application of the RG transformation for the lattice with inhomogeneous bonds, where two types of cells are taken into account, makes it possible to investigate the behavior of the specific heat in a relatively wide range of temperatures.

Our modification of the RG method proposed by Stella et al. has been fruitful with respect to the preservation of the Hamiltonian symmetry. The eigenvalues of the so-called "parity" operator are better suited for splitting the cell eigenvectors than the sign of the eigenvalues of the total spin $z$ component. The "parity" symmetry has proven to be essential and should be conserved during the RG transformation.

We report that the low-temperature peak is consistently present by comparing the data from zeroth- and first-order calculations. The position of the peak depends on the value of the bond ratio $J_{L} / J_{K}$. As this ratio goes down, the peak shifts toward zero temperature. Although in both zeroth- and first-order approximations the low-temperature maximum disappears in the pure kagomé lattice, we do not exclude that in reality it could survive in a higher-order approximation.

\section{ACKNOWLEDGMENTS}

We wish to thank Professor A.L. Stella and Professor J. Sznajd for discussions and M. Dudziński for stimulating remarks. One of us (A.D.) would like to thank the K.U. Leuven for warm hospitality and support from the K.U. Leuven Research Fund (F/95/21).
* On leave of absence from the Institute for Low Temperature and Structure Research, Polish Academy of Sciences, P.O. Box 937, 50-950 Wroclaw 2, Poland.

${ }^{1}$ S.R. White and R.M. Noack, Phys. Rev. Lett. 68, 3487 (1992); S.R. White, ibid. 69, 2863 (1992); S.R. White, Phys. Rev. B 48, 10345 (1993).

${ }^{2}$ S.D. Drell, M. Weinstein, and S. Yankielowicz, Phys. Rev. D 14, 487 (1976).

${ }^{3}$ K. Penson, R. Jullien, and P. Pfeuty, Phys. Rev. B 22, 380 (1980).

${ }^{4}$ L.S. Campana, A. Caramico D'Auria, U. Esposito, and G. Ka- mieniarz, J. Phys. C 20, 5161 (1987).

${ }^{5}$ A. Drzewiński and J.M.J. van Leeuwen, Phys. Rev. B 49, 403 (1994).

${ }^{6}$ A. Drzewiński and R. Dekeyser, Phys. Rev. B 51, 15218 (1995).

${ }^{7}$ R.J. Bursill and F. Gode, J. Phys. Condens. Matter 7, 9765 (1995).

${ }^{8}$ A.L. Stella and F. Toigo, Physica A 89, 175 (1977).

${ }^{9}$ A.L. Stella, C. Vanderzande, and R. Dekeyser, Phys. Rev. B 27, 1812 (1983).

${ }^{10}$ N. Elstner, R.R.P. Singh, and A.P. Young, Phys. Rev. Lett. 71, 
1629 (1993), and references therein.

${ }^{11}$ R.R.P. Singh and D. Huse, Phys. Rev. Lett. 68, 1766 (1992).

${ }^{12}$ P.W. Leung and V. Elser, Phys. Rev. B 47, 5459 (1993).

${ }^{13}$ D.S. Greywall and P.A. Busch, Phys. Rev. Lett. 62, 1868 (1989).

${ }^{14}$ V. Elser, Phys. Rev. Lett. 62, 2405 (1989).

${ }^{15}$ N. Elstner and A.P. Young, Phys. Rev. B 50, 6871 (1994), and references therein.

${ }^{16}$ T. Nakamura and S. Miyashita, Phys. Rev. B 52, 9174 (1995).

${ }^{17}$ K. Fukamachi and H. Nishimori, Phys. Rev. B 49, 651 (1994).

${ }^{18}$ A. Drzewiński and J. Sznajd, Phase Transit. 57, 1 (1996).

${ }^{19}$ P. Pfeuty, R. Jullien, and K.A. Penson, in Real-Space Renormal- ization, Topics in Current Physics Vol. 30, edited by T.W. Burkhardt and J.M.J. van Leeuwen (Springer-Verlag, Berlin, 1982).

${ }^{20}$ J. Sznajd (private communication).

${ }^{21}$ J.Th. Niemeijer and J.M.J. van Leeuwen, Physica A 71, 17 (1974).

${ }^{22}$ J.E. Hirsch and J.V. José, Phys. Rev. B 22, 5339 (1980); J.E. Hirsch, ibid. 22, 5355 (1980).

${ }^{23}$ H. Franco, R.E. Rapp, and H. Godfrin, Phys. Rev. Lett. 57, 1161 (1986). 